\title{
Synthesis of Folic Acid-Modified DOX@ZIF-8 Nanoparticles for Targeted Therapy of Liver Cancer
}

\author{
Jian Bi, ${ }^{1}$ Yishan Lu, ${ }^{2}$ Yan Dong $\left(\mathbb{D},{ }^{3}\right.$ and Ping Gao $\mathbb{D}^{3}$ \\ ${ }^{1}$ Department of Gastroenterology, The First Affiliated Hospital of Dalian Medical University, Dalian 116011, China \\ ${ }^{2}$ Queen Mary College, Nanchang University, Nanchang, Jiangxi 330031, China \\ ${ }^{3}$ Department of Oncology, The First Affiliated Hospital of Dalian Medical University, Dalian 116011, China
}

Correspondence should be addressed to Yan Dong; dongyan100@aliyun.com and Ping Gao; gaopinggaoping2008@163.com

Received 14 March 2018; Revised 6 June 2018; Accepted 28 June 2018; Published 30 July 2018

Academic Editor: Ilaria Armentano

Copyright (c) 2018 Jian Bi et al. This is an open access article distributed under the Creative Commons Attribution License, which permits unrestricted use, distribution, and reproduction in any medium, provided the original work is properly cited.

\begin{abstract}
The effective chemotherapy treatment for liver cancer patients remains an urgent issue due to the difficulty in precisely delivering drugs to the tumor site. The targeted delivery of drugs by nanoparticles is a promising strategy to address this problem. However, the fabrication of drug targeted delivery nanosystem still remains a major challenge. In this study, a novel folic acid-functionalized (doxorubicin, DOX) DOX@ZIF-8 nanoparticles (DOX@ZIF-8-FA) were prepared as a liver cancer-targeted drug delivery system. The delivery nanosystem exhibited a high drug loading capacity $(15.7 \mathrm{wt} \%)$ and presented excellent drug-sustained release performances and good pH-responsive properties. Compared with free DOX and DOX@ZIF-8 nanoparticles, the DOX@ZIF-8FA nanoparticles displayed much higher anticancer efficiency in HepG2 cells, suggesting that the folic acid-functionalized DOX@ZIF-8 nanoparticles have promising applications in targeted treatment of cancer cells.
\end{abstract}

\section{Introduction}

Hepatocellular carcinoma (HCC) is the major form of liver cancer and is the third most common cause of cancer death worldwide [1]. Currently, chemotherapy is the major treatment method. However, advanced HCC often has a poor prognosis, and only a few chemotherapeutic drugs, such as sorafenib, demonstrate efficacy in increasing overall survival in advanced or metastatic HCC [2]. Due to the rapid development of drug resistance and low targeting ability, the effective course of chemotherapy for HCC often lasts for only a few months [3]. Thus, developing new strategies for targeted drug delivery and controllable drug release is vital.

With the rapid development of nanotechnology, nanoscaled materials such as mesoporous silica nanomaterials, metal nanoparticles, polymers, liposomes, and metalorganic-framework (MOF) materials [4-9] are used as drug vehicles for reducing side effects and enhancing therapeutic efficacy, which offers an alternative strategy to address the problem discussed above. Among these nanocarriers, zeolitic imidazolate framework (ZIF), a subclass of MOF, with large surface areas, high porosity, and high stability, has been extensively utilized in drug delivery. Recently, several studies showed that ZIF-8 possessed a remarkable drug loading capacity and a pH-triggered control of drug release property [10-16]. However, a major drawback is their lack of tumor targeting ability. Further study is needed to develop tumor targeting ligand-functionalized MOFs to achieve targeted drug delivery and enhance therapeutic efficacy.

In this study, we report the synthesis of a folic acidmodified (doxorubicin, DOX) DOX@ZIF-8 nanoparticles (abbreviated as DOX@ZIF-8-FA) for targeted treatment of human hepatocellular carcinoma HepG2 cells. The DOX@ZIF-8-FA nanoparticles were characterized by scanning electron microscopy (SEM), transmission electron microscopy (TEM), powder X-ray diffraction (PXRD), and zeta potential meter. Their in vitro cytotoxicity and therapeutic efficacy in HepG2 cells were also investigated using MTT assays. Furthermore, we demonstrated that the DOX@ZIF-8-FA complex has a higher anticancer efficiency towards the HepG2 cell lines compared with doxorubicin in the absence of ZIF- 
8 nanoparticles. The proliferation of HepG2 cells was significantly decreased after DOX@ZIF-8-FA treatment.

\section{Materials and Methods}

2.1. Materials and Chemicals. Zinc nitrate hexahydrate $\left(\mathrm{Zn}\left(\mathrm{NO}_{3}\right)_{2} \cdot 6 \mathrm{H}_{2} \mathrm{O}\right)$, folic acid $(\mathrm{FA})$, and ethanol were obtained from Sinopharm Chemical Reagent Co. Ltd. (Shanghai, China). 2-Methylimidazole was purchased from Alfa Aesar. Doxorubicin (DOX) was purchased from Huafeng United Technology Co. Ltd. (Beijing, China). The other chemicals in this experiment were of analytical grade and used without further purification.

2.2. Synthesis of DOX@ZIF-8 Nanoparticles. The DOX@ZIF8 nanoparticles were prepared by optimizing the previously reported method [10]. In brief, $1.0 \mathrm{~mL}$ of DOX solution (DOX, $12 \mathrm{mg}$ ) was mixed with $200 \mu \mathrm{L}$ of zinc nitrate solution $\left(\mathrm{Zn}\left(\mathrm{NO}_{3}\right)_{2} \cdot 6 \mathrm{H}_{2} \mathrm{O}, 50 \mathrm{mg}\right)$. The mixture was shaken at room temperature for $2 \mathrm{~min}$. Then, $2.5 \mathrm{~g}$ of an aqueous solution containing $0.5 \mathrm{~g}$ of 2 -methylimidazole was added dropwise to the above solution under ultrasonication. Following 15 min ultrasonication, the DOX@ZIF-8 nanoparticles were collected, washed with ethanol and water, and then dispersed in water. The quantification of DOX loaded in ZIF-8 nanoparticles was measured by using UV/Vis spectroscopy with the absorbance at $479 \mathrm{~nm}$. The supernatant was used to quantify the DOX concentration. Because the obtained DOX@ZIF-8 nanoparticles are insoluble in the aqueous solution, the percentage of loaded amount of DOX was determined by performing the subtraction of the amounts of their initial and remaining DOX in the aqueous solution. The quantification of DOX was performed using the calibration curve of DOX in phosphate buffer $(\mathrm{pH}=7.4$, Supplementary Figure S1). The loading amount was calculated as follows:

$$
\begin{aligned}
& \text { Loading amount }(\%) \\
& =\frac{\mathrm{DOX}_{\text {added }}(\mathrm{mg})-\mathrm{DOX}_{\text {remained }}(\mathrm{mg})}{\mathrm{DOX} @ \mathrm{ZIF}-8_{\text {total }}(\mathrm{mg})} * 100 \%
\end{aligned}
$$

2.3. Synthesis of FA-Modified DOX@ZIF-8 (DOX@ZIF-8-FA) Nanoparticles.20 mg of DOX@ZIF-8 nanoparticles was dispersed in $1 \mathrm{~mL}$ of water, followed by adding $1.0 \mathrm{~mL}$ of FA solution (FA, $20 \mathrm{mg} / \mathrm{mL}, \mathrm{pH}=7.0$ ). The mixture was applied to ultrasonic treatment at room temperature for $10 \mathrm{~min}$. The obtained DOX@ZIF-8-FA nanoparticles were separated by centrifugation, washed with ethanol and water, and dissolved in water for further use.

2.4. In Vitro DOX Release from the DOX@ZIF-8-FA Nanoparticles. DOX release from the DOX@ZIF-8-FA nanoparticles analysis was performed in PBS buffer solution $\left(\mathrm{pH}=7.4,6.0\right.$, and 5.0 , resp.) at $37^{\circ} \mathrm{C}$. For each release study, $200 \mu \mathrm{g}$ of DOX@ZIF-8-FA nanoparticles was dispersed in $200 \mu \mathrm{L}$ of PBS buffer solution and incubated at $37^{\circ} \mathrm{C}$. At a given time, the nanoparticles were collected by centrifugation; $200 \mu \mathrm{L}$ of fresh PBS buffer solution was added for the next release experiment. The DOX content in the release solution was measured by an UV/Vis spectrophotometry

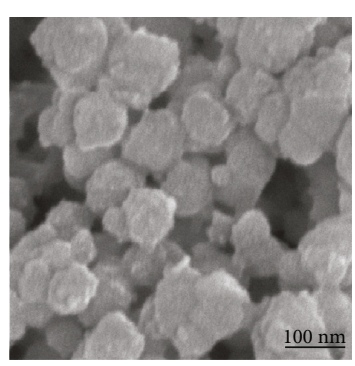

(a)

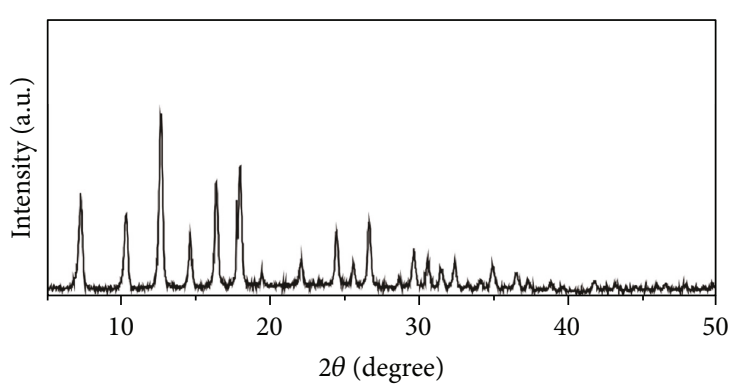

(c)

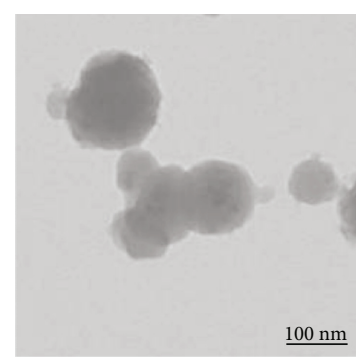

(b)
FIgURE 1: SEM image (a), TEM image (b), and XRD patterns (c) of DOX@ZIF-8 nanoparticles.

with the absorbance at $479 \mathrm{~nm}$. The released percentage of DOX was calculated by the ratio of the released amount of DOX to the total loaded amount of DOX.

2.5. Cell Culture and Proliferation Assays. Cells were maintained in DMEM (supplemented with $10 \%$ fetal bovine serum and $1 \%$ antibiotic/antimycotic agent) at $37^{\circ} \mathrm{C}$ in $5 \%$ $\mathrm{CO}_{2}$ and $21 \% \mathrm{O}_{2}$. HepG2 cells were plated at a concentration of $5 \times 10^{4}$ cells $/ \mathrm{mL}$ in a 24 -well plate and were treated with $25 \mathrm{nM}$ free DOX, DOX@ZIF-8 nanoparticles, or DOX@ZIF-8-FA nanoparticles. Cell viability assays were performed using a 3-(4,5-dimethylthiazol-2-yl)-2,5-diphenyltetrazolium bromide (MTT) reagent as previously described [17].

2.6. Statistical Analysis. Statistical analyses were performed by the Student $t$-test (two-tailed) using Prism GraphPad software. Differences with $P<0.05$ were considered statistically significant. Data were represented as mean \pm SEM.

\section{Results and Discussion}

High-dispersion DOX@ZIF-8 nanoparticles were synthesized using the modified method developed by Zheng et al. (please see experimental section for details) [10]. As seen in SEM and TEM images (Figures 1(a) and 1(b)), the DOX@ZIF-8 nanoparticles are nearly spherical with a very coarse surface. The particle size was also measured from SEM images at three different regions. The data revealed that the obtained DOX@ZIF-8 nanoparticles have a wide particle size distribution with a diameter of $30-110 \mathrm{~nm}$, which is smaller than Zheng and coworkers' reported results [10]. This might be due to ultrasonication which could accelerate the reaction rate and produce more seed crystals at the early stage of the 
TABLE 1: Zeta potential of DOX@ZIF-8 nanoparticles and DOX @ ZIF-8-FA nanoparticles in ultrapure water.

\begin{tabular}{lc}
\hline Samples & Zeta potential $(\mathrm{mV})$ \\
\hline DOX@ZIF-8 nanoparticles & $+28.0 \pm 0.2$ \\
DOX@ZIF-8-FA nanoparticles & $-4.9 \pm 0.1$ \\
\hline
\end{tabular}

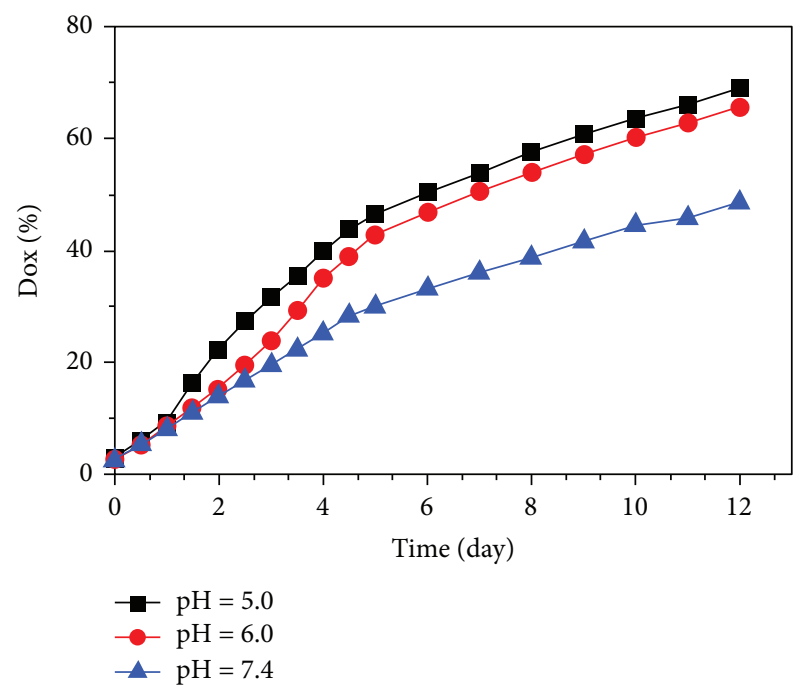

Figure 2: Release kinetics of DOX from DOX@ZIF-8-FA nanoparticles in PBS buffer solution at different $\mathrm{pH}$ values.

reaction, leading to the decrease of the particle sizes. The powder X-ray diffraction (PXRD) pattern of the DOX@ZIF-8 nanoparticles is shown in Figure 1(c). All of the sharp diffraction peaks can be indexed to the crystalline ZIF-8 particles, and no other peaks of impurity were observed in the PXRD patterns, suggesting high purity of the obtained DOX@ZIF-8 nanomaterials.

In order to endow this drug delivery system with tumor targeted ability, the DOX@ZIF-8 nanoparticles were integrated with folic acid, which has been widely used as a targeting ligand for treatment of folate receptor-overexpressing tumors including breast, lung, liver, and ovarian cancers $[18,19]$. The surface functionalization of the particles was then investigated by zeta potential measurements. As revealed in Table 1, the zeta potential of the DOX@ZIF-8 nanoparticles and the DOX@ZIF-8-FA nanoparticles was $+28.0 \mathrm{mV}$ and $-4.9 \mathrm{mV}$, respectively. The zeta potential of the DOX@ZIF-8-FA nanoparticles was lower than the DOX@ZIF-8 nanoparticles, which was due to the present of negative charged carboxyl groups on the nanoparticles' surface by folic acid functionalization.

The release profiles of DOX from the DOX@ZIF-8-FA nanoparticles with $15.7 \mathrm{wt} \%$ DOX loading in different $\mathrm{pH}$ values of PBS buffer are shown in Figure 2. There was only $48.5 \%$ of DOX released during the test time in PBS buffer at $\mathrm{pH} 7.4$, whereas the cumulative release of DOX was about $68.8 \%$ at $\mathrm{pH} 5.0$ and $65.5 \%$ at $\mathrm{pH} 6.0$, respectively, in 12 days, suggesting that the DOX@ZIF-8-FA drug delivery system possesses excellent drug-sustained release performances and good $\mathrm{pH}$-responsive properties, which are similar to

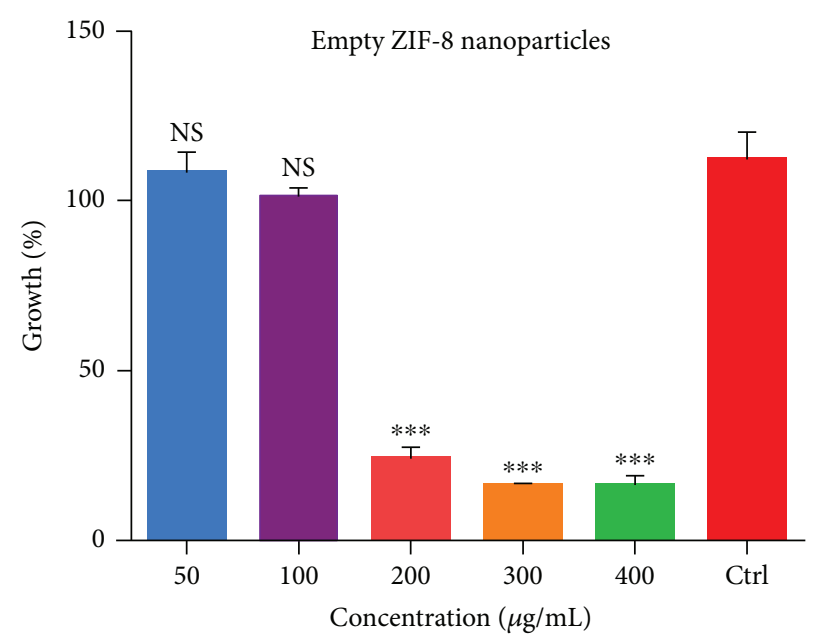

FIGURE 3: In vitro cytotoxicity of empty ZIF-8 nanoparticles against HepG2 cells at different concentrations after $24 \mathrm{~h} .{ }^{* * *} P<0.001$ versus control. NS: No statistical significance versus control.

previously reported DOX-ZIF-8 drug delivery nanosystems $[10,12]$. The $\mathrm{pH}$-responsive drug release mechanism of the DOX@ZIF-8-FA nanosystem involves two possible factors $[12,20]$ : (1) the easy degradation of ZIF-8 nanoparticles in acidic environments and (2) the increased solubility of DOX at low $\mathrm{pH}$ due to the increased protonation of amino groups in DOX molecules.

It is very important to evaluate the biocompatibility of the ZIF-8 NPs for their potential bioapplications. Hence, we performed the standard 3-(4,5-dimethylthiazol-2-yl)-2,5diphenyltetrazolium bromide (MTT) assay on HepG2 cells to investigate the cytotoxicity of the ZIF-8 nanoparticles. Figure 3 revealed the cytotoxic efficacy of the ZIF-8 NPs with the HepG2 cells. The results demonstrated that the ZIF-8 NPs have minimal toxicity at lower concentration and can be applied in the biomedical field with good biocompatibility.

Finally, we have investigated the roles of DOX@ZIF-8FA nanoparticles in the progression of the HCC cell line. The results showed that DOX@ZIF nanoparticles or DOX@ZIF-8-FA nanoparticles significantly inhibited the growth of HepG2 cells compared to equivalent concentration of free DOX alone (Figure 4). Moreover, DOX@ZIF-8-FA nanoparticles exerted a better inhibition function than the treatment with either DOX@ZIF nanoparticles at the DOX concentration of $7.5 \mu \mathrm{g} / \mathrm{mL}$ for $24 \mathrm{~h}$ (Figure 4 ). The concentrations causing $70 \%$ cell growth inhibition. The possible mechanism may be attributed to the targeting ligand (folic acid) on the particle surface to enhance cellular uptake of the nanoparticles into HepG2 cells, resulting a higher therapeutic effect. In addition, our results have shown that the cell morphology was destroyed after treated with DOX@ZIF nanoparticles or DOX@ZIF-8-FA nanoparticles compared to free DOX (Supplementary Figure S2).

\section{Conclusions}

In conclusion, we have reported a novel folic acid-modified DOX@ZIF-8 nanoparticles for targeted treatment of liver 


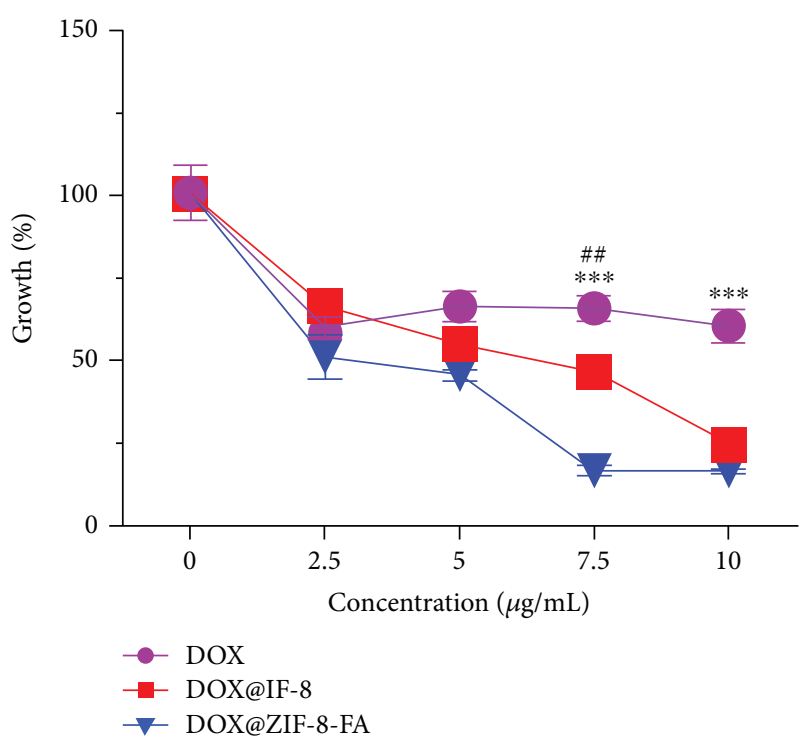

FIGURE 4: MTT assay in HepG2 cells treated with DOX, DOX@ZIF8 nanoparticles, or DOX@ZIF-8-FA nanoparticles at the equivalent concentration of free DOX after $24 \mathrm{~h} .{ }^{* * *} P<0.001$ versus DOX, \#\# $P<0.01$ versus DOX@ZIF-8.

cancer. Doxorubicin hydrochloride (DOX), selected as a model drug, was efficiently entrapped in ZIF-8 nanoparticles. The low cytotoxicity, high drug capacity, and tumor targeting ability make it a promising targeted drug delivery system. MTT assay also showed that DOX@ZIF-8-FA nanoparticles displayed a higher therapeutic efficiency towards HepG2 cells than free DOX and DOX@ZIF-8 nanoparticles. These results indicated that the DOX@ZIF-8-FA nanoparticles are a promising drug delivery system for folate receptor-related liver cancer.

\section{Data Availability}

The data used to support the findings of this study are available from the corresponding author upon request.

\section{Conflicts of Interest}

The authors declare that they have no competing interests.

\section{Authors' Contributions}

Jian Bi and Ping Gao designed the experiments. Jian Bi, Yishan Lu, and Yan Dong performed the experiments. Jian Bi, Yan Dong, and Ping Gao analyzed the data. Jian Bi, Yan Dong, and Ping Gao contributed to the writing of the manuscript. Jian Bi, Yishan Lu, Yan Dong, and Ping Gao revised the manuscript. All authors reviewed the manuscript.

\section{Acknowledgments}

This work was supported by Natural Science Foundation of Liaoning Province, China (no. 20162225), and Education Department of Liaoning Province project (no. L2016019).

\section{Supplementary Materials}

Supplementary Figure S1: the calibration curve of DOX in PBS solution. Supplementary Figure S2: the cell morphology was destroyed after treated with DOX@ZIF nanoparticles, DOX@ZIF-8-FA nanoparticles, and free DOX. (Supplementary Materials)

\section{References}

[1] A. Jemal, F. Bray, M. M. Center, J. Ferlay, E. Ward, and D. Forman, "Global cancer statistics," CA: A Cancer Journal for Clinicians, vol. 61, no. 2, pp. 69-90, 2011.

[2] B. Xie, D. H. Wang, and S. J. Spechler, "Sorafenib for treatment of hepatocellular carcinoma: a systematic review," Digestive Diseases and Sciences, vol. 57, no. 5, pp. 1122-1129, 2012.

[3] H. Y. Kim and J. W. Park, "Molecularly targeted therapies for hepatocellular carcinoma: sorafenib as a stepping stone," Digestive Diseases, vol. 29, no. 3, pp. 303-309, 2011.

[4] Z. Li, J. C. Barnes, A. Bosoy, J. F. Stoddart, and J. I. Zink, "Mesoporous silica nanoparticles in biomedical applications," Chemical Society Reviews, vol. 41, no. 7, pp. 2590-2605, 2012.

[5] P. Ghosh, G. Han, M. De, C. K. Kim, and V. M. Rotello, "Gold nanoparticles in delivery applications," Advanced Drug Delivery Reviews, vol. 60, no. 11, pp. 1307-1315, 2008.

[6] M. S. Yavuz, Y. Cheng, J. Chen et al., "Gold nanocages covered by smart polymers for controlled release with near-infrared light,” Nature Materials, vol. 8, no. 12, pp. 935-939, 2009.

[7] A. Kumari, S. K. Yadav, and S. C. Yadav, "Biodegradable polymeric nanoparticles based drug delivery systems," Colloids and Surfaces B: Biointerfaces, vol. 75, no. 1, pp. 1-18, 2010.

[8] P. Horcajada, T. Chalati, C. Serre et al., "Porous metalorganic-framework nanoscale carriers as a potential platform for drug delivery and imaging," Nature Materials, vol. 9, no. 2, pp. 172-178, 2010.

[9] M.-X. Wu and Y.-W. Yang, "Metal-organic framework (MOF)-based drug/cargo delivery and cancer therapy," Advanced Materials, vol. 29, no. 23, article 1606134, 2017.

[10] H. Zheng, Y. Zhang, L. Liu et al., "One-pot synthesis of metalorganic frameworks with encapsulated target molecules and their applications for controlled drug delivery," Journal of the American Chemical Society, vol. 138, no. 3, pp. 962-968, 2016.

[11] H. Ren, L. Zhang, J. An et al., “Polyacrylic acid@zeolitic imidazolate framework-8 nanoparticles with ultrahigh drug loading capability for $\mathrm{pH}$-sensitive drug release," Chemical Communications, vol. 50, no. 8, pp. 1000-1002, 2014.

[12] C.-Y. Sun, C. Qin, X.-L. Wang et al., "Zeolitic imidazolate framework-8 as efficient $\mathrm{pH}$-sensitive drug delivery vehicle," Dalton Transactions, vol. 41, no. 23, pp. 6906-6909, 2012.

[13] I. B. Vasconcelos, T. G. da Silva, G. C. G. Militão et al., "Cytotoxicity and slow release of the anti-cancer drug doxorubicin from ZIF-8," RSC Advances, vol. 2, no. 25, pp. 9437-9442, 2012.

[14] X. Wang, D. Miao, X. Liang et al., "Nanocapsules engineered from polyhedral ZIF-8 templates for bone-targeted hydrophobic drug delivery," Biomaterials Science, vol. 5, no. 4, pp. 658662, 2017.

[15] E. Shearier, P. Cheng, Z. Zhu, J. Bao, Y. H. Hu, and F. Zhao, "Surface defection reduces cytotoxicity of $\mathrm{Zn}(2$-methylimidazole $)_{2}$ (ZIF-8) without compromising its drug delivery capacity," RSC Advances, vol. 6, no. 5, pp. 4128-4135, 2016. 
[16] X. Cao, L. Dai, L. Wang, J. Liu, and J. Lei, "A surfactant template-assisted strategy for synthesis of ZIF-8 hollow nanospheres," Materials Letters, vol. 161, pp. 682-685, 2015.

[17] H. Zhang, S. K. Ramakrishnan, D. Triner et al., "Tumorselective proteotoxicity of verteporfin inhibits colon cancer progression independently of YAP1," Science Signaling, vol. 8, no. 397, p. ra98, 2015.

[18] J. Sudimack and R. J. Lee, “Targeted drug delivery via the folate receptor," Advanced Drug Delivery Reviews, vol. 41, no. 2, pp. 147-162, 2000.

[19] Y. G. Assaraf, C. P. Leamon, and J. A. Reddy, "The folate receptor as a rational therapeutic target for personalized cancer treatment," Drug Resistance Updates, vol. 17, no. 4-6, pp. 89-95, 2014.

[20] Z. Liu, X. Sun, N. Nakayama-Ratchford, and H. Dai, "Supramolecular chemistry on water-soluble carbon nanotubes for drug loading and delivery," ACS Nano, vol. 1, no. 1, pp. 5056, 2007. 


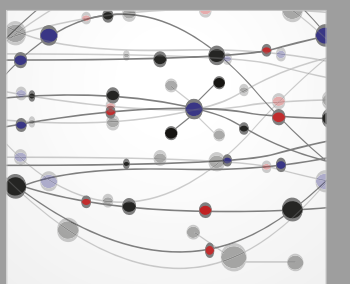

The Scientific World Journal
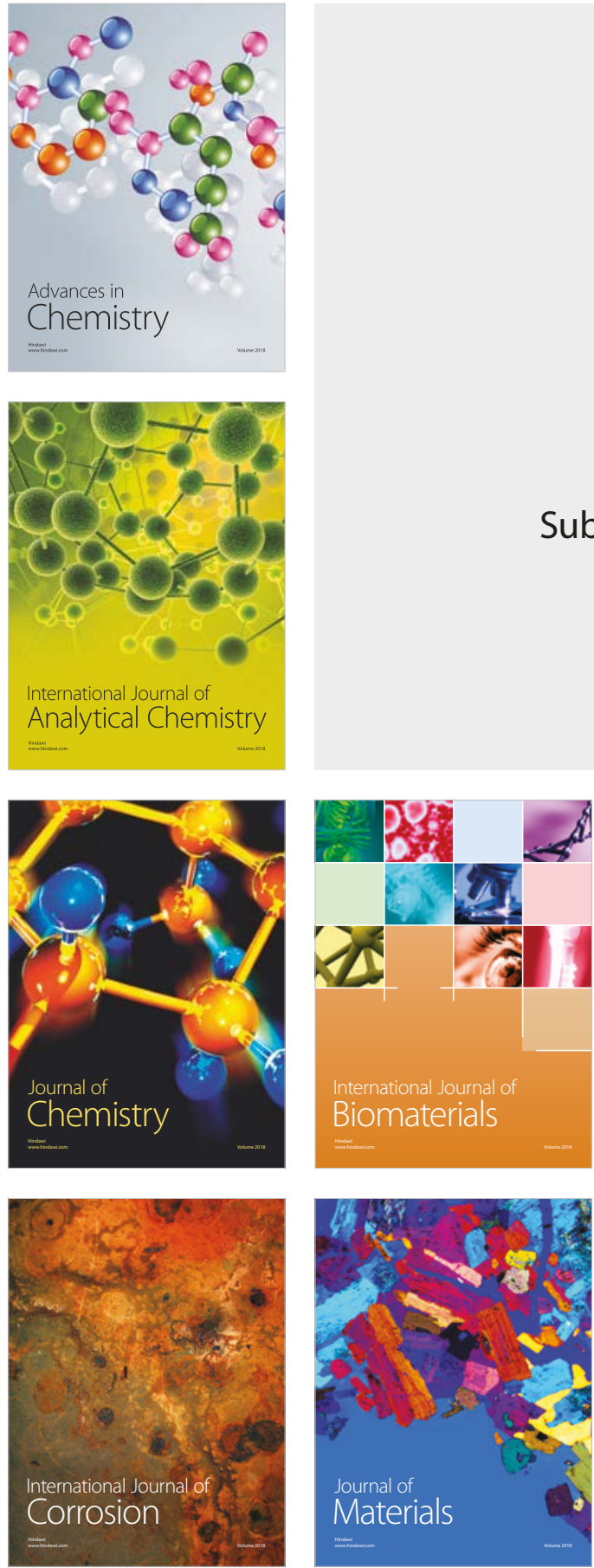

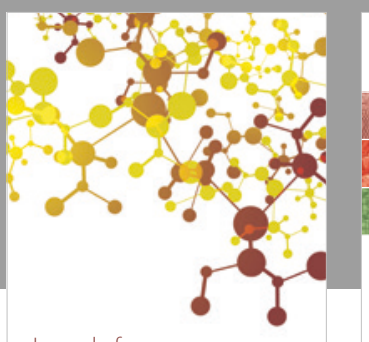

Journal of

Applied Chemistry
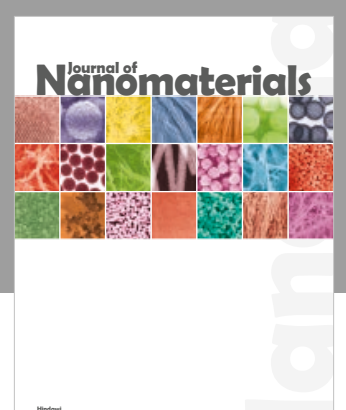

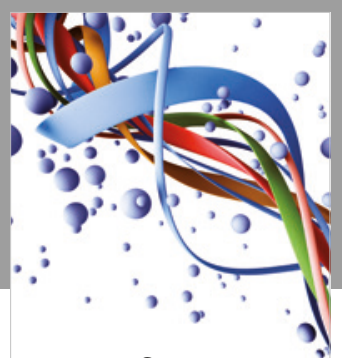

Scientifica

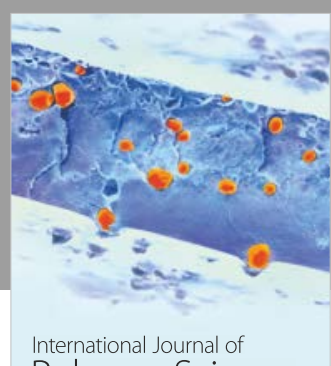

Polymer Science

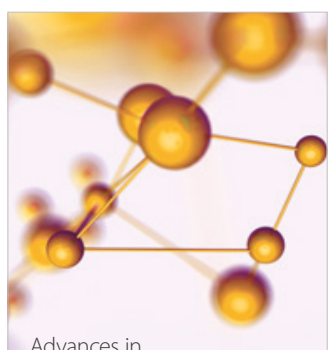

Physical Chemistry
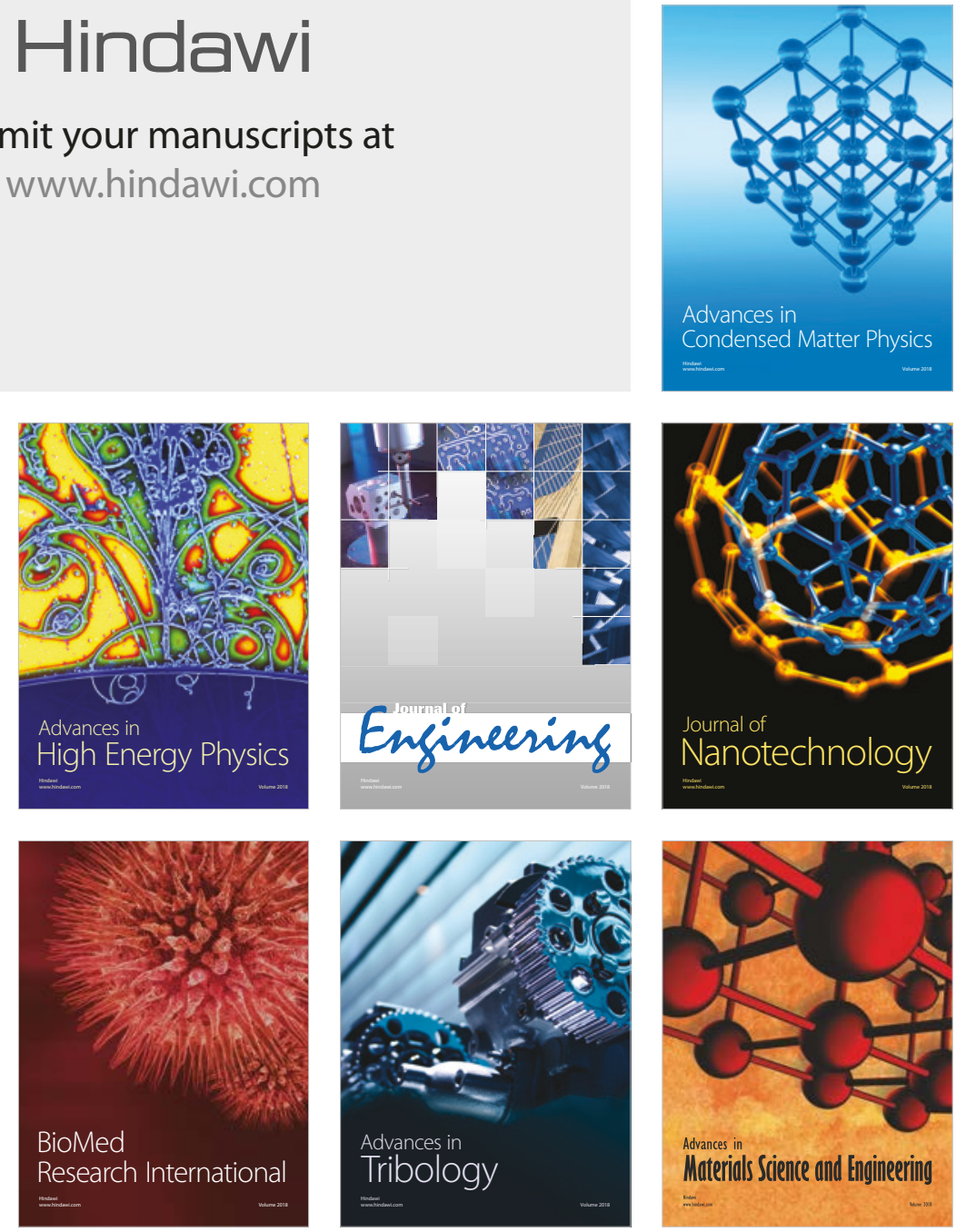